Annals of Pure and Applied Mathematics

Vol. 15, No. 2, 2017, 327-339

ISSN: 2279-087X (P), 2279-0888(online)

Published on 11 December 2017

www.researchmathsci.org

DOI: http://dx.doi.org/10.22457/apam.v15n2a19

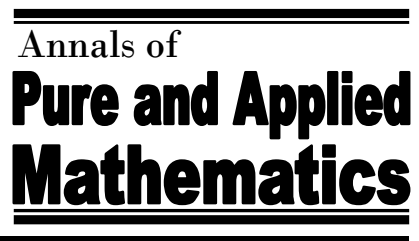

\title{
Inventory Model for Deteriorating Items with no Shortages
}

\author{
P. Mariappan ${ }^{1}$, M. Kameswari ${ }^{2}$ and M. Antony Raj ${ }^{3}$ \\ ${ }^{1}$ Research Supervisor in Mathematics, Bishop Heber College \\ Tiruchirappalli-620017, India. E-mail: mathmari@yahoo.com \\ ${ }^{2}$ Department of Mathematics, Thiagarajar College of Engineering \\ Tamilnadu, Madurai - 55, India. \\ ${ }^{3}$ Department of Actuarial Science, Bishop Heber College, \\ Tiruchirappalli - 17, Tamilnadu, Madurai - 55, India.
}

Received 20 November 2017; accepted 9 December 2017

\begin{abstract}
This paper develops a lot-sizing model for deteriorating items by allowing the demand and the deterioration time to be stochastic and follows an exponential distribution. The formula for the essential parameters are derived. The model is tested with the empirical data and the results are furnished.
\end{abstract}

Keywords: Inventory, deteriorating items, economic ordering quantity, optimum cost

AMS Mathematics Subject Classification (2010): 90B05

\section{Introduction}

Inventory is simply a stock of physical goods (assets) having certain economic values, which can be in any one the following forms namely material, money or labor. It may be considered as those commodities which are secured, stored and used in a day-to-day functioning of the system. In the past, inventories were also viewed as measures of the wealth and power of an individual of a country based on the amount of wheat, sold etc. Stored in its warehouses. During this period managing the inventories was a comfortable matter. The control and maintenance of production inventories of deteriorating items with no shortages have attracted much attention in inventory analysis because; most of the physical goods are deteriorating over the time period. The issue of deterioration is very significant in many inventory systems. Deterioration is defined as decay or damage such that the items cannot be used for its original purpose. Food items, drugs, pharmaceuticals, radioactive substances are examples of items in which sufficient deterioration can take office during the normal storage period of the units and consequently this loss must be taken into account when analyzing the system.

\section{Review of literature}

All the relevant review of literature collected by the researcher based on the suggested title is listed below year wise. (Samanta and Roy, 2004) state that a continuous production control inventory model of deteriorating items with shortages. It is assumed that the production rate is changed to another at a time when the inventory level reaches a 


\section{P.Mariappan, M.Kameswari and M.Antony Raj}

prefixed level Q1 and continued until the inventory level reaches the level $\mathrm{S}\left(>\mathrm{Q}_{1}\right)$. The demand rate is assumed to be constant. The production is started again at a time when the shortage level reaches a prefixed quantity $\mathrm{Q}_{2}$. (Samanta and Roy, 2004) developed a continuous production control inventory model for deteriorating items with shortages is developed. A number of structural properties of the inventory system are studied analytically. Here the demand rate and production rate are constant and the distribution of the time to deterioration of an item follows the exponential distribution. The main focus is on the structural behavior of the system. (Hesham Alfares, 2007) deals an inventory model in which the demand rate depends on the inventory level are based on the common real-life observation that greater product availability tends to stimulate more sales. Previous models incorporating inventory-level dependent demand rate assume that the holding cost is constant for the entire inventory cycle and they consider the inventory policy for an item with a stock-level dependent demand rate and a storage-time dependent holding cost. The holding cost per unit of the item per unit time is assumed to be an increasing function of the time spent in storage. Two time-dependent holding cost step functions are considered: Retroactive holding cost increase, and incremental holding cost increase. Procedures are developed for determining the optimal order quantity and the optimal cycle time for both cost structures. (Khanra and Chaudhur, 2011) is developed a production-inventory model over a finite planning horizon where the demand varies linearly with time. The machine production rate is assumed to be finite and constant. Shortages in inventory are allowed and are completely backlogged. (KuoHou, Huang and Lin, 2011)presents an inventory model for deteriorating items with stock-dependent selling rate under inflation and time value of money over a finite planning horizon. In the model, shortages are allowed and the unsatisfied demand is partially backlogged at the exponential rate with respect to the waiting time. They establish the theoretical results and provide an efficient solution procedure to find the optimal number of replenishment and the cycle time. Then, the optimal order quantity and the total present value of profits are obtained. (Sachin Kumar, Pawan Kumar and Manju Saini, 2012) seeks a deterministic inventory model has been developed for deteriorating items. Demand is taken as quadratic functions of time. Shortages are not allowed and the effect of inflation rate in this model for deteriorating items under inflation. The mathematical expression for the total relevant cost and other parameter has been obtained. (Maragatham and Lakshmidevi, 2013) discussed an inventory model to find the minimum relevant inventory cost per unit time for non-instantaneous deteriorating goods over a finite time horizon with exponentially declining demand for $\mathrm{n}$ cycles. The shortages are allowed and back ordered. Under the situation of delay in payments, the inventory model in this study is divided into four cases by the time of shortage, deadline of delay in payment. (Dash, Singh and Pattnayak, 2014) calculated a total optimal cost of an inventory model with exponential declining demand and constant deterioration is considered. The time-varying holding cost is a linear function of time. Shortages are not allowed. The items (like food grains, fashion apparels and electronic equipment) have fixed shelf-life which decreases with time during the end of the season. (Kumar, Vasishtha and Gupta, 2014) states a production control inventory model for continuous deteriorating items with shortages. Here they assumed that the production rate is changed to another at a time when the inventory level reaches a prefixed level $\mathrm{Q}_{1}$ and continued until the inventory level reaches the level $\mathrm{S}\left(\mathrm{Q}_{1}\right)$ and the demand rate as 


\section{Inventory Model for Deteriorating Items with no Shortages}

constant. The production is started again at a time when the shortage level reaches a prefixed quantity $\mathrm{Q}_{2}$. In this model the total cost per unit time as a function of $\mathrm{Q}_{1}, \mathrm{Q}_{2}$ and $\mathrm{S}$ is derived. (Rathod and Bhathawala, 2014) proposed the inventory models in which the demand rate depends on the inventory level are based on the common real-life observation that greater product availability tends to stimulate more sales. Previous models incorporating stock-level dependent demand rate assumes that the holding cost is constant for the entire inventory cycle. They discussed a stock-level dependent demand rate and a storage-time dependent holding cost and also the variable ordering cost. The holding cost per unit of the item per unit time is assumed to be an increasing function of the time spent in storage. Two time-dependent holding cost step functions are considered: Retroactive holding cost increase, and incremental holding cost increase. (Pal and Chandra, 2014) states a periodic review inventory model with stock dependent demand. When stock on hand is zero, the inventory manager offers a price discount to customers who are willing to backorder their demand. Permissible delay in payments allowed to the inventory manager is also taken into account. (Sivashankari and Panayappan, 2014) seeks the inventory model in production scheduling and planning. The EPQ model is commonly used by practitioners in the fields of production and inventory management to assist them in making decisions on optimum production and total cost. The total cost of production is dependent on production rate, demand rate, and rate of deteriorating items. A inventory model with deteriorative items, in which two different rates of productions are considered, and it is possible that production started at one rate, and after some time, it may be switched over another rate such a situation is desirable in the sense that by starting at a low rate of production, a large quantum stock of manufacturing items at the initial stage is avoided, leading to a reduction in the holding cost. The variation in production rate provides a way resulting consumer satisfaction and earning potential profit. (Lakshmi Devi and Maragatham, 2015) considered an inventory model with three different rates of production and quadratic demand rate. The shortages are allowed and deterioration rate is time dependent. (Kaliraman, Chandra and Chaudhry, 2015) developed an economic production quantity inventory model for deteriorating items; the rate of deterioration is Waybill distribution deterioration with two parameters. The rate of demand is stock dependent. Shortages are not allowed. The aim of this study is to find the optimal solution for minimizing the total inventory costs. (Sharma, 2015) has discussed about Permissible delay in payment basically termed as "Trade credit financing" and in the present business scenario it has become a popular trend to attract the retailers by offering some interest free period by suppliers/wholesalers to increase the sales. The phenomenon of trade credit has drawn attention of many researches for last decay. Here the author considered this phenomenon under some conditions with combination of constant demand and holding cost. Shortages are permitted in the OW and partially backlogged at the next replenishment cycle. The objective of modeling is to derive the retailer's optimal replenishment policy that minimizes the total relevant inventory cost per unit of time. This model dealt with a single item. (Sharmila and Uthayakumar, 2016) is considered an inventory model with three different rates of production rate and stock dependent demand. Deterioration plays a vital role in the present environment of the market. In this paper, a two parameter Waybill distribution has been used to represent the deterioration rate. The objective is to determine the optimal total cost and the optimal time schedule of the plan for the proposed model. (Patel, 2017) has explained a 


\section{P.Mariappan, M.Kameswari and M.Antony Raj}

deteriorating item production inventory model with time and price dependent demand under inflation and permissible delay. Different deterioration rates are considered in a cycle. Shortages are allowed. In this paper, the researcher developed a lot-sizing model for deteriorating items by allowing the demand and the deterioration time be stochastic and follows Exponential distribution. The formula for the essential parameters were derived. The model is tested with the empherical data and the results are furnished.

\section{Notations and assumptions}

The following notations and assumptions are used for developing the model.

(i) $\quad \mathrm{A}(>0)$ is initial demand and $\lambda$ is constant.

(ii) $\quad \mathrm{p}(>\mathrm{a})$ is the constant production rate

(iii) $\quad C_{1}$ is the holding cost per unit per unit time

(iv) $\quad C_{2}$ is the cost of a deteriorated unit $\left[C_{1}, C_{2}\right.$ Are known constant $]$

(v) $\mathrm{C}$ is the total inventory cost (or) the average system cost

(vi) $\mathrm{Q}(\mathrm{t})$ is the inventory level at time $\mathrm{t}(\geq 0)$

(vii) $\mathrm{g}(\mathrm{t})$ is the inventory deteriorates per unit time

(viii) $\mathrm{R}(\mathrm{t})$ is the demand rate per unit time

(ix) Replenishment is instantaneous and lead time is zero

(x) $\quad \mathrm{T}$ is the fixed duration of a production cycle $\left(t_{1}\right.$ is time interval)

(xi) The distribution of the time to deterioration of an item follows the exponential distribution $\mathrm{g}(\mathrm{t})$ where

$$
g(t)=\left\{\begin{array}{cl}
\theta e^{-\theta t}, & \text { for } t>0 \\
0, & \text { otherwise }
\end{array}\right.
$$

$\theta$ is called the deterioration rate a constant fraction $\theta(0<\theta \ll 1)$ of the on Inventory deteriorates per unit time

(xii) The demand rate is known and decreases exponentially.

$$
R(t)=\left\{\begin{array}{rr}
A e^{-\lambda t} & Q(t)>0 \\
0 & \text {,otherwise }
\end{array}\right.
$$

where $\mathrm{A}(>0)$ is the initial demand and $\lambda,(0<\lambda<\theta)$ is a constant.

This model is the represented by the following diagram

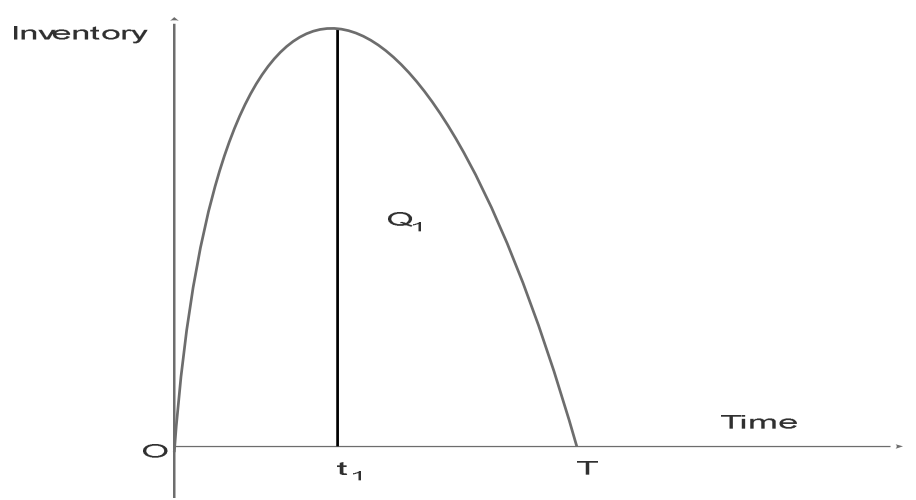

Figure 1: 
Inventory Model for Deteriorating Items with no Shortages

Production starts at time $\mathrm{t}=0$ and stop at $\mathrm{t}=\mathrm{t}_{1}$. During $\left[0, \mathrm{t}_{1}\right]$, the production rate is $\mathrm{p}$ and demand rate is a $(<p)$. The stock attains a level $q_{1}$ at time $t=t_{1}$. Hence there is no shortages accumulate. Again the production starts at rate $\mathrm{p}$ at $\mathrm{t}=\mathrm{t}_{1}$. The cycle then repeats itself after time t.

\section{The mathematical model and its analysis}

Let $\mathrm{Q}(\mathrm{t})$ be the on-hand inventory at time $\mathrm{t}(0 \leq t \leq T)$ then the differential equations governing instantaneous state of $\mathrm{Q}(\mathrm{t})$ at any time $\mathrm{t}$ are given by

$$
\begin{array}{ll}
\frac{d Q(t)}{d t}+\theta Q(t)=p-A e^{-\lambda t} & , 0 \leq t \leq t_{1} \\
\frac{d Q(t)}{d t}+\theta Q(t)=-A e^{-\lambda t} & , t_{1} \leq t \leq T
\end{array}
$$

The boundary conditions are

$$
\mathrm{Q}(0)=0, \mathrm{Q}\left(\mathrm{t}_{1}\right)=\mathrm{Q}_{1}, \mathrm{Q}(\mathrm{T})=0
$$

The solutions of equation $(1) \Rightarrow$

$$
\begin{gathered}
\frac{d Q(t)}{d t}+\theta Q(t)=p-A e^{-\lambda t \quad} \quad 0 \leq t \leq t_{1} \\
p=\theta, \quad Q=p-A e^{-\lambda t}, \quad Y=Q(t) \\
Q(t) e^{\int \theta d t}=\int\left(p-A e^{-\lambda t}\right) e^{\int \theta d t}+C \\
Q(t) e^{\theta t}=\int\left(p-A e^{-\lambda t}\right) e^{\theta t}+C \\
Q(t) \mathrm{e}^{\theta \mathrm{t}}=\frac{\mathrm{pe}^{\theta \mathrm{t}}}{\theta}-\frac{\mathrm{Ae}^{(\theta-\lambda) t}}{\theta-\lambda}+\mathrm{c} \\
Q(t) e^{\theta t}=\int\left(p e^{\theta t}-A e^{(\theta-\lambda) t}\right) d t+C \\
Q(\mathrm{t})=\frac{\mathrm{p}}{\theta}-\frac{A \mathrm{e}^{(\theta-\lambda) \mathrm{t}}}{(\theta-\lambda) \mathrm{e}^{\theta \mathrm{t}}}+\frac{\mathrm{C}}{\mathrm{e}^{\theta \mathrm{t}}} \\
\mathrm{Q}(\mathrm{t})=\frac{p}{\theta}-\frac{A e^{-\lambda t}}{\theta-\lambda}+\frac{C}{e^{\theta t}}
\end{gathered}
$$

Using boundary condition

$\mathrm{Q}(0)=0$

$$
\begin{aligned}
\Rightarrow Q(0)=\frac{p}{\theta}- & \frac{A}{\theta-\lambda}+C \\
& =\frac{p}{\theta}-\frac{A}{\theta-\lambda}+C ; C=\frac{A}{\theta-\lambda}-\frac{p}{\theta}
\end{aligned}
$$

$\mathrm{C}$ in (4)

$$
\begin{gathered}
Q(t)=\frac{p}{\theta}-\frac{A e^{-\lambda t}}{\theta-\lambda}+\frac{1}{e^{\theta t}}\left(\frac{A}{\theta-\lambda}-\frac{p}{\theta}\right)=\frac{\mathrm{p}}{\theta}-\frac{A \mathrm{e}^{-\lambda \mathrm{t}}}{\theta-\lambda}+\frac{\mathrm{A}}{\mathrm{e}^{\theta \mathrm{t}}(\theta-\lambda)}-\frac{\mathrm{p}}{\theta \mathrm{e}^{\theta \mathrm{t}}} \\
\mathrm{Q}=\frac{\mathrm{p}}{\theta}\left(1-\mathrm{e}^{-\theta \mathrm{t}}\right)+\frac{\mathrm{A}}{\theta-\lambda}\left(\mathrm{e}^{-\theta \mathrm{t}}-\mathrm{e}^{-\lambda \mathrm{t}}\right)
\end{gathered}
$$

$$
\begin{gathered}
(2) \Rightarrow \quad \frac{\mathrm{dQ}(\mathrm{t})}{\mathrm{dt}}+\theta \mathrm{Q}(\mathrm{t})=-\mathrm{Ae}^{-\lambda \mathrm{t}}, \quad t_{1} \leq \mathrm{t} \leq \mathrm{T} \\
Y=Q(\mathrm{t}), \quad p=Q, \quad Q=-A e^{-\lambda t} \\
\mathrm{Q}(\mathrm{t}) \mathrm{e}^{\int \theta \mathrm{dt}}=\int-\mathrm{Ae}^{-\lambda \mathrm{t}} \cdot \mathrm{e}^{\int \theta \mathrm{dt}} \mathrm{dt}+\mathrm{C} \\
\mathrm{Q}(\mathrm{t}) \mathrm{e}^{\theta \mathrm{t}}=\int-\mathrm{Ae}^{-\lambda \mathrm{t}} \cdot \mathrm{e}^{\theta \mathrm{t}} \mathrm{dt}+\mathrm{C}
\end{gathered}
$$


P.Mariappan, M.Kameswari and M.Antony Raj

$$
\begin{aligned}
& \mathrm{Q}(\mathrm{t}) \mathrm{e}^{\theta \mathrm{t}}=-\int \mathrm{A} e^{(\theta-\lambda) t} \mathrm{dt}+\mathrm{C} \\
& \mathrm{Q}(\mathrm{t}) \mathrm{e}^{\theta \mathrm{t}}=-\frac{\mathrm{A} e^{(\theta-\lambda) t}}{(\theta-\lambda)}+C \\
& \mathrm{Q}(\mathrm{t})=-\frac{\mathrm{A} e^{(\theta-\lambda) t}}{\mathrm{e}^{\theta \mathrm{t}}(\theta-\lambda)}+\frac{C}{\mathrm{e}^{\theta \mathrm{t}}} \\
& \mathrm{Q}(\mathrm{t})=-\frac{A}{(\theta-\lambda)} e^{-\lambda t}+\frac{C}{\mathrm{e}^{\theta \mathrm{t}}}
\end{aligned}
$$

Using boundary conditions

$\mathrm{Q}\left(\mathrm{t}_{1}\right)=\mathrm{Q}_{1}$

$$
\begin{gathered}
\mathrm{Q}\left(t_{1}\right)=-\frac{A}{(\theta-\lambda)} e^{-\lambda t_{1}}+\frac{C}{\mathrm{e}^{\theta t_{1}}} \\
Q_{1}=-\frac{A}{(\theta-\lambda)} e^{-\lambda t_{1}}+\frac{C}{\mathrm{e}^{\theta t_{1}}} \\
C=Q_{1} e^{\theta t_{1}}+\frac{A}{(\theta-\lambda)} e^{\theta t_{1}} \cdot e^{-\lambda t_{1}}=Q_{1} e^{\theta \mathrm{t}_{1}}+\frac{\mathrm{A}}{(\theta-\lambda)} \mathrm{e}^{(\theta-\lambda) \mathrm{t}_{1}} \\
\mathrm{Q}(\mathrm{t})=-\frac{\mathrm{A}}{\theta-\lambda} \mathrm{e}^{-\lambda \mathrm{t}_{1}}+\frac{1}{\mathrm{e}^{\theta \mathrm{t}}}\left[\mathrm{Q}_{1} \mathrm{e}^{\theta \mathrm{t}_{1}}+\frac{\mathrm{A}}{\theta-\lambda} \mathrm{e}^{(\theta-\lambda) \mathrm{t}_{1}}\right] \\
=-\frac{\mathrm{A}}{\theta-\lambda} \mathrm{e}^{-\lambda \mathrm{t}_{1}}+\mathrm{Q}_{1} \mathrm{e}^{\theta\left(\mathrm{t}_{1}-\mathrm{t}\right)}+\frac{\mathrm{A}}{(\theta-\lambda) \mathrm{e}^{\theta \mathrm{t}}} \mathrm{e}^{(\theta-\lambda) \mathrm{t}_{1}} \\
=\frac{A}{\theta-\lambda}\left[\frac{e^{(\theta-\lambda) t_{1}}}{e^{\theta t}}-e^{-\lambda t}\right]+Q_{1} e^{\theta\left(t_{1}-t\right)} \\
\mathrm{Q}(\mathrm{t})=\frac{\mathrm{A}}{\theta-\lambda}\left[\frac{\mathrm{e}^{(\theta-\lambda) \mathrm{T}_{1}-\mathrm{e}^{(\theta-\lambda) \mathrm{t}}}}{\mathrm{e}^{\theta \mathrm{t}}}\right]+\mathrm{Q}_{1} \mathrm{e}^{\theta\left(\mathrm{t}_{1}-\mathrm{t}\right)}, \quad t_{1} \leq \mathrm{t} \leq \mathrm{T}
\end{gathered}
$$

From (1)-(2),

$$
Q(t)= \begin{cases}\frac{\mathrm{p}}{\theta}\left(1-\mathrm{e}^{-\theta \mathrm{t}}\right)+\frac{\mathrm{A}}{\theta-\lambda}\left(\mathrm{e}^{-\theta \mathrm{t}}-\mathrm{e}^{-\lambda \mathrm{t}}\right), & 0 \leq t \leq t_{1} \\ \frac{\mathrm{A}}{\theta-\lambda}\left[\frac{\mathrm{e}^{(\theta-\lambda) \mathrm{T}_{1}}-\mathrm{e}^{(\theta-\lambda) \mathrm{t}}}{\mathrm{e}^{\theta \mathrm{t}}}\right]+\mathrm{Q}_{1} \mathrm{e}^{\theta\left(\mathrm{t}_{1}-\mathrm{t}\right)}, & t_{1} \leq t \leq T\end{cases}
$$

From (3) and (5)

$$
\begin{aligned}
& \begin{array}{l}
(3) \Rightarrow \quad Q\left(t_{1}\right)=Q_{1} \\
(5) \Rightarrow
\end{array} \\
& \quad Q(t)=\frac{\mathrm{p}}{\theta}\left(1-\mathrm{e}^{-\theta t_{1}}\right)+\frac{\mathrm{A}}{\theta-\lambda}\left(\mathrm{e}^{-\theta t_{1}}-\mathrm{e}^{-\lambda t_{1}}\right) ; \quad Q\left(t_{1}\right)=Q_{1} \\
& \quad=\frac{\mathrm{p}}{\theta}\left(1-\mathrm{e}^{-\theta t_{1}}\right)+\frac{\mathrm{A}}{\theta-\lambda}\left(\mathrm{e}^{-\theta t_{1}}-\mathrm{e}^{-\lambda t_{1}}\right)
\end{aligned}
$$


Inventory Model for Deteriorating Items with no Shortages

$$
\begin{gathered}
\quad=p t_{1}-A t_{1} \\
Q_{1}=t_{1}[p-A]=t_{1}\left[p-\frac{A}{\theta-\lambda}(\theta-\lambda)\right] ; \quad Q_{1}=t_{1}[p-A] \\
t_{1}=\frac{Q_{1}}{p-A}, \quad 0 \leq t \leq t_{1}
\end{gathered}
$$

Again from (3) and (7), we have

$$
\begin{gathered}
(3) \Rightarrow \quad Q(T)=0 \\
\begin{array}{c}
(7) \Rightarrow \quad Q(t)=\frac{A}{\theta-\lambda}\left[\frac{e^{(\theta-\lambda) t_{1}}-e^{(\theta-\lambda) t}}{e^{\theta t}}\right]+Q_{1} e^{\theta\left(t_{1}-t\right)} \\
0=Q(T)=-\frac{A}{\theta-\lambda}\left(\frac{e^{(\theta-\lambda) t_{1}}}{e^{\theta T}}\right)+\frac{A}{\theta-\lambda}\left(\frac{e^{(\theta-\lambda) T}}{e^{\theta T}}\right) \\
Q_{1} e^{\theta t_{1}}=-\frac{A}{\theta-\lambda}\left(e^{(\theta-\lambda) t_{1}}\right)+\frac{A}{\theta-\lambda}\left(e^{(\theta-\lambda) T}\right) \\
Q_{1}\left(1+\theta t_{1}\right)=-\left(\frac{A}{\theta-\lambda}+\frac{A}{\theta-\lambda} t_{1}(\theta-\lambda)\right)+\frac{A}{\theta-\lambda}+\frac{A}{\theta-\lambda} T(\theta-\lambda) \\
=\frac{A}{\theta-\lambda}(\theta-\lambda)\left[T-t_{1}\right] \\
Q_{1}\left(1+\theta t_{1}\right)=A\left[T-t_{1}\right] \\
Q_{1}\left(1+\theta t_{1}\right)+A t_{1}=A T \\
\quad T=\frac{Q_{1}}{A}\left(1+\theta t_{1}\right)+t_{1}
\end{array}
\end{gathered}
$$

Using (8)

$$
T=\frac{Q_{1}}{A}\left(1+\frac{\theta Q_{1}}{p-A}\right)+\frac{Q_{1}}{p-A}
$$

\section{Therefore, total deterioration in [0, T]}

$$
\begin{aligned}
& =\left\{\left(p-A e^{-\lambda t_{1}}\right) t_{1}-Q_{1}\right\}+\left\{Q_{1}-A e^{-\lambda\left(T-t_{1}\right)}\right\} \\
& =\left\{\left(p-A e^{-\lambda t_{1}}\right) t_{1}-A e^{-\lambda\left(T-t_{1}\right)} \cdot\left(T-t_{1}\right)\right\} \\
& =p t_{1}-A e^{-\lambda t_{1}} \cdot t_{1}-A e^{-\lambda\left(T-t_{1}\right)} \cdot\left(T-t_{1}\right) \\
& =\frac{p Q_{1}}{p-A}-A\left(\frac{Q_{1}}{p-A}\right)\left(1-\lambda t_{1}\right)-\left(A T-A t_{1}\right)\left(1-\lambda\left(T-t_{1}\right)\right) \\
& =\frac{p Q_{1}}{p-A}-\frac{A Q_{1}}{p-A}+\frac{A \lambda Q_{1}}{p-A}-A\left(T-t_{1}\right)+A \lambda\left(T-t_{1}\right)^{2} \\
& =\frac{p Q_{1}}{p-A}-\frac{A Q_{1}}{p-A}+\frac{A \lambda Q_{1}}{p-A}-A\left[T-\frac{Q_{1}}{p-A}\right]+A \lambda\left[T^{2}-\frac{2 T Q_{1}}{p-A}+\frac{Q_{1}^{2}}{(p-A)^{2}}\right] \\
=\frac{\mathrm{pQ}}{\mathrm{p}-\mathrm{A}} & -\frac{A Q_{1}}{p-A}+\frac{A \lambda Q_{1}}{p-A}-A T+\frac{A Q_{1}}{p-A}+A \lambda T^{2}-\frac{2 T A \lambda Q_{1}}{p-A}+\frac{A \lambda Q_{1}^{2}}{(p-A)^{2}} \\
(\mathrm{Neglecting} \mathrm{higher} \mathrm{power} \mathrm{of} \theta) & \frac{(p-A) Q_{1}}{(p-A)}+\frac{A \lambda Q_{1}}{p-A}-A T+\frac{A Q_{1}}{p-A}+A \lambda T^{2}-\frac{2 T A \lambda Q_{1}}{p-A}+\frac{A \lambda Q_{1}^{2}}{(p-A)^{2}}
\end{aligned}
$$

The deterioration cost over the period $[0, T]$

$$
=C_{2}\left\{Q_{1}\left[1+\frac{A \lambda}{p-A}+\frac{A}{p-A}-\frac{2 T A \lambda}{p-A}\right]+Q_{1}^{2}\left[\frac{A \lambda}{(p-A)^{2}}\right]-A T+A \lambda T^{2}\right\}
$$


P.Mariappan, M.Kameswari and M.Antony Raj

The inventory carrying cost over the cycle $[0, T]$

$$
\begin{array}{r}
=C_{1} \int_{0}^{T} Q(t) d t=C_{1}\left[\int_{0}^{T} Q(t) d t+\int_{t_{1}}^{T} Q(t) d t\right] \\
=C_{1}\left[\int_{0}^{t_{1}}\left(\frac{p}{\theta}\left(1-e^{-\theta t}\right)+\frac{A}{\theta-\lambda}\left(e^{-\theta t}-e^{-\lambda t}\right)\right) d t\right] \\
+\int_{t_{1}}^{T} \frac{A}{\theta-\lambda}\left(\frac{e^{(\theta-\lambda) t_{1}}-e^{(\theta-\lambda) t}}{e^{\theta t}}\right)+Q_{1} e^{\theta\left(t_{1}-t\right)} d t
\end{array}
$$

LHS$$
\int_{0}^{t_{1}} \frac{p}{\theta}\left(1-e^{-\theta t}\right)+\frac{A}{\theta-\lambda}\left(e^{-\theta t}-e^{-\lambda t}\right)
$$$$
=\int_{0}^{t_{1}} \frac{p}{\theta}\left(1-\left(1-\theta t+\frac{\theta^{2} t^{2}}{2 !}\right)\right)
$$$$
+\frac{A}{\theta-\lambda}\left(1-\theta t+\frac{\theta^{2} t^{2}}{2 !}-1+\lambda t-\frac{\lambda^{2} t^{2}}{2 !}\right) d t
$$$$
=\int_{0}^{t_{1}} \frac{p}{\theta}\left(\theta t+\frac{\theta^{2} t^{2}}{2 !}\right)+\frac{A}{\theta-\lambda}(\lambda t-\theta t) d t
$$

(Neglecting higher power of $\theta$ )

$$
\begin{gathered}
=\left[\int_{\theta}^{t_{1}}\left(\frac{p}{\theta} \theta t\right)-\frac{A}{\theta-\lambda}(\theta-\lambda) t d t\right]=\left[\int_{0}^{t}(p t-A t) d t\right] \\
=\left(\frac{p t^{2}}{2}-\frac{A t^{2}}{2}\right)_{0}^{t_{1}} \\
=\frac{p t_{1}^{2}}{2}-\frac{A t_{1}^{2}}{2} \quad(a) \\
\int_{0}^{t_{1}} \frac{p}{\theta}\left(1-e^{-\theta t}\right)+\frac{A}{\theta-\lambda}\left(e^{-\theta t}-e^{-\lambda t}\right) d t=\frac{t_{1}^{2}}{2}(p-A) \\
R H S \quad \int_{t_{1}}^{T} \frac{A}{\theta-\lambda}\left(\frac{e^{(\theta-\lambda) t_{1}}-e^{(\theta-\lambda) t}}{\left.\int^{\theta t}\right)+Q_{1} e^{\theta\left(t_{1}-t\right)} d t}\right. \\
=\int_{t_{1}}^{T} \frac{A}{\theta-\lambda} e^{(\theta-\lambda) t_{1}-\theta t}-\frac{A}{\theta-\lambda} e^{-\lambda t}+Q_{1} e^{\theta\left(t_{1}-t\right)} d t \\
=\left[-\frac{A}{\theta-\lambda} \frac{1}{\theta} e^{(\theta-\lambda) t_{1}-\theta t}+\frac{1}{\lambda}\left(\frac{A}{\theta-\lambda}\right) e^{-\lambda t}-\frac{Q_{1}}{\theta} e^{\theta\left(t_{1}-t\right)}\right]_{t_{1}}^{T} \\
=-\frac{1}{\theta}\left(\frac{A}{\theta-\lambda}\right) e^{(\theta-\lambda) t_{1}-\theta T}+\frac{1}{\lambda}\left(\frac{A}{\theta-\lambda}\right) e^{-\lambda T}-\frac{Q_{1}}{\theta} e^{\theta\left(t_{1}-T\right)} \\
\quad+\frac{A}{\theta-\lambda} \frac{1}{\theta} e^{(\theta-\lambda) t_{1}-\theta t_{1}}-\frac{1}{\lambda}\left(\frac{\mathrm{A}}{\theta-\lambda}\right) \mathrm{e}^{-\lambda \mathrm{t}_{1}}+\frac{\mathrm{Q}_{1}}{\theta} \mathrm{e}^{\left(\mathrm{t}_{1}-\mathrm{t}_{1}\right)} \\
=\frac{\mathrm{A}}{\theta(\theta-\lambda)} \mathrm{e}^{-\lambda \mathrm{t}_{1}}\left[\mathrm{e}^{\left(\mathrm{t}_{1}-\mathrm{T}\right)}-1\right]-\frac{\mathrm{Q}_{1}}{\theta}\left[\mathrm{e}^{\theta\left(\mathrm{t}_{1}-\mathrm{T}\right)}-1\right] \\
+\frac{1}{\lambda} \frac{\mathrm{A}}{\theta-\lambda}\left[\mathrm{e}^{-\lambda \mathrm{T}}-\mathrm{e}^{-\lambda \mathrm{t}_{1}}\right]
\end{gathered}
$$


Inventory Model for Deteriorating Items with no Shortages

$$
\begin{aligned}
& =\left[\mathrm{e}^{\theta\left(\mathrm{t}_{1}-\mathrm{T}\right)}-1\right]\left[-\frac{\mathrm{A}}{\theta(\theta-\lambda)} \mathrm{e}^{-\lambda \mathrm{t}_{1}}-\frac{\mathrm{Q}_{1}}{\theta}\right]+\frac{1}{\lambda} \frac{\mathrm{A}}{\theta-\lambda}\left[\mathrm{e}^{-\lambda \mathrm{T}}-\mathrm{e}^{\lambda \mathrm{t}_{1}}\right] \\
& =\left[1+\theta\left(t_{1}-T\right)\right. \\
& \ldots \ldots \ldots \ldots-1]\left[-\frac{A}{\theta(\theta-\lambda)}\left\{1-\lambda t_{1}\right\}-\frac{Q_{1}}{\theta}\right] \\
& +\frac{1}{\lambda} \frac{A}{\theta-\lambda}\left[1-\lambda T+\frac{\lambda^{2} T^{2}}{2 !}+\ldots \ldots \ldots \ldots-1+\lambda t_{1}\right. \\
& \left.-\frac{\lambda^{2} t_{1}^{2}}{2 !}\right]
\end{aligned}
$$

(Neglecting higher power of $\theta$ )

$$
\begin{aligned}
&= {\left[1+\theta\left(t_{1}-T\right) \ldots \ldots \ldots-1\right]\left[\frac{-A}{\theta(\theta-\lambda)}\left\{1-\lambda t_{1}\right\}-\frac{Q_{1}}{\theta}\right] } \\
& \quad+\frac{1}{\lambda} \frac{A}{\theta-\lambda}\left[\lambda\left(t_{1}-T\right)\right] \\
&= \theta\left(t_{1}-T\right)\left\{\frac{-A}{\theta(\theta-\lambda)}+\frac{A \lambda t_{1}}{\theta(\theta-\lambda)}-\frac{Q_{1}}{\theta}\right\}+\frac{A}{\theta-\lambda}\left(t_{1}-T\right) \\
&= \frac{-\left(t_{1}-T\right) A}{\theta-\lambda}+\frac{\left(t_{1}-T A \lambda t_{1}\right)}{\theta-\lambda}-\left(t_{1}-T\right) Q_{1}+\frac{A}{\theta-\lambda}\left(t_{1}-T\right) \\
&=\left(t_{1}-T\right)\left[\frac{-A}{\theta-\lambda}+\frac{A \lambda t_{1}}{\theta-\lambda}-Q_{1}+\frac{A}{\theta-\lambda}\right] \\
&=\left(t_{1}-T\right)\left[\frac{A \lambda t_{1}}{\theta-\lambda}-Q_{1}\right] \\
&= {\left[\frac{Q_{1}}{p-A}-T\right]\left[\frac{A \lambda t_{1}}{\theta-\lambda}-Q_{1}\right] } \\
&=\frac{Q_{1}^{2} A \lambda}{(\theta-\lambda)(p-A)^{2}}-\frac{Q_{1}^{2}}{p-A}-\frac{T A \lambda Q_{1}}{(\theta-\lambda)(p-A)}+Q_{1} T
\end{aligned}
$$

Therefore, the inventory carrying cost over the cycle $[0, \mathrm{~T}]$ is

$$
\begin{aligned}
& \Rightarrow C_{1}\left\{\frac{t_{1}^{2}}{2}(p-A)+\frac{Q_{1}^{2} A \lambda}{(\theta-\lambda)(p-A)^{2}}-\frac{Q_{1}^{2}}{p-A}-\frac{T A \lambda Q_{1}}{(\theta-\lambda)(p-A)}+Q_{1} T\right\} \\
& \Rightarrow C_{1}\left\{\frac{Q_{1}^{2}}{2(p-A)}-\frac{Q_{1}^{2} A \lambda}{(\theta-\lambda)(p-A)^{2}}-\frac{Q_{1}^{2}}{(p-A)}-\frac{T A \lambda Q_{1}}{(\theta-\lambda)(p-A)}+Q_{1} T\right\}
\end{aligned}
$$

Hence the total inventory cost of the system

To find stock level $\left(Q_{1}^{*}\right)$ :

$$
\begin{gathered}
C^{*}=\frac{C_{1}}{T}\left\{\frac{Q_{1}^{2}}{2(p-A)}-\frac{Q_{1}^{2} A \lambda}{(\theta-\lambda)(p-A)^{2}}-\frac{Q_{1}^{2}}{(p-A)}-\frac{Q_{1} A T \lambda}{(\theta-\lambda)(p-A)}+Q_{1} T\right\} \\
+\frac{\mathrm{C}_{2}}{\mathrm{~T}}\left\{\mathrm{Q}_{1}\left(1+\frac{\mathrm{A} \lambda}{\mathrm{p}-\mathrm{A}}+\frac{\mathrm{A}}{\mathrm{p}-\mathrm{A}}-\frac{2 \mathrm{TA} \lambda}{\mathrm{p}-\mathrm{A}}\right)\right\}+\mathrm{Q}_{1}^{2}\left(\frac{\mathrm{A} \lambda}{(\mathrm{p}-\mathrm{A})^{2}}\right) \\
-\mathrm{AT}+\mathrm{A}^{2} \mathrm{~T}^{2}
\end{gathered}
$$

$$
\frac{d C\left(Q_{1}\right)}{d Q_{1}}=0
$$


P.Mariappan, M.Kameswari and M.Antony Raj

$$
\begin{gathered}
\frac{d C\left(Q_{1}\right)}{d Q_{1}}=\left\{\frac{C_{1} Q_{1}}{T(p-A)}-\frac{2 Q_{1} A \lambda C_{1}}{T(\theta-\lambda)(p-A)^{2}}-\frac{2 Q_{1} C_{1}}{T(p-A)}-\frac{A \lambda C_{1}}{(\theta-\lambda)(p-A)}+C_{1}\right\} \\
+\left\{\frac{C_{2}}{T}+\frac{C_{2} A \lambda}{T(p-A)}+\frac{A C_{2}}{T(p-A)}-\frac{2 A \lambda C_{2}}{(p-A)}+\frac{2 Q_{1} A \lambda C_{2}}{(p-A)}\right\}=0
\end{gathered}
$$

$Q_{1}^{*}$ is the solution of the equation $\frac{d C\left(Q_{1}\right)}{d Q_{1}}=0$

$$
\begin{aligned}
Q_{1}^{*}=\left(A \lambda C_{1}(p\right. & -A) T-C_{1} T(p-A)^{2}(\theta-\lambda)-C_{2}(p-A)^{2}(\theta-\lambda) \\
& -C_{2} A \lambda(p-A)(\theta-\lambda)-A C_{2}(p-A)(\theta-\lambda) \\
& \left.+2 A \lambda C_{2}(p-A)(\theta-\lambda) T\right) /\left(C_{1}(\theta-\lambda)(p-A)-2 A \lambda C_{1}\right. \\
& \left.-2 C_{1}(\theta-\lambda)(p-A)-2 A \lambda C_{2} T(p-A)(\theta-\lambda)\right)
\end{aligned}
$$

To find average $\operatorname{cost}\left(\mathrm{C}^{*}\right)$ :

$$
\begin{gathered}
C^{*}=\frac{C_{1}}{T}\left\{\frac{Q_{1}^{2}}{2(p-A)}-\frac{Q_{1}^{2} A \lambda}{(\theta-\lambda)(p-A)^{2}}-\frac{Q_{1}^{2}}{(p-A)}-\frac{Q_{1} A T \lambda}{(\theta-\lambda)(p-A)}+Q_{1} T\right\} \\
+\frac{\mathrm{C}_{2}}{\mathrm{~T}}\left\{\mathrm{Q}_{1}\left(1+\frac{\mathrm{A} \lambda}{\mathrm{p}-\mathrm{A}}+\frac{\mathrm{A}}{\mathrm{p}-\mathrm{A}}-\frac{2 \mathrm{TA} \lambda}{\mathrm{p}-\mathrm{A}}\right)\right\}+\mathrm{Q}_{1}^{2}\left(\frac{\mathrm{A} \lambda}{(\mathrm{p}-\mathrm{A})^{2}}\right)-\mathrm{AT} \\
+\mathrm{A} \lambda \mathrm{T}^{2}
\end{gathered}
$$

\section{Numerical illustration and its analysis numerical example}

Here we have calculated optimal stock level $Q_{1}^{*}$, and the minimum average system cost $C^{*}$ for given value of production cycle length $T$ and other parameters by considering the example.

Example 1. Let $\theta=0.1, c_{1}=4, c_{2}=20, P=20, A=2$, and $T=80$ in appropriate units.

$$
\begin{gathered}
\mathrm{Q}_{1}^{*}=\left(\mathrm{A} \lambda \mathrm{C}_{1}(\mathrm{p}-\mathrm{A}) \mathrm{T}-\mathrm{C}_{1} \mathrm{~T}(\mathrm{p}-\mathrm{A})^{2}(\theta-\lambda)-\mathrm{C}_{2}(\mathrm{p}-\mathrm{A})^{2}(\theta-\lambda)\right. \\
-\mathrm{C}_{2} \mathrm{~A} \lambda(\mathrm{p}-\mathrm{A})(\theta-\lambda)-\mathrm{AC}_{2}(\mathrm{p}-\mathrm{A})(\theta-\lambda) \\
\left.+2 \mathrm{~A} \lambda \mathrm{C}_{2}(\mathrm{p}-\mathrm{A})(\theta-\lambda) \mathrm{T}\right) /\left(\mathrm{C}_{1}(\theta-\lambda)(\mathrm{p}-\mathrm{A})-2 \mathrm{~A} \lambda \mathrm{C}_{1}\right. \\
\left.-2 \mathrm{C}_{1}(\theta-\lambda)(\mathrm{p}-\mathrm{A})-2 \mathrm{~A} \lambda \mathrm{C}_{2} \mathrm{~T}(\mathrm{p}-\mathrm{A})(\theta-\lambda)\right) \\
\boldsymbol{Q}_{1}^{*}=\mathbf{9 . 3 4 6 6} \\
C^{*}=\frac{C_{1}}{T}\left\{\frac{Q_{1}^{2}}{2(p-A)}-\frac{Q_{1}^{2} A \lambda}{(\theta-\lambda)(p-A)^{2}}-\frac{Q_{1}^{2}}{(p-A)}-\frac{Q_{1} A T \lambda}{(\theta-\lambda)(p-A)}+Q_{1} T\right\} \\
+\frac{\mathrm{C}_{2}}{\mathrm{~T}}\left\{\mathrm{Q}_{1}\left(1+\frac{\mathrm{A} \lambda}{\mathrm{p}-\mathrm{A}}+\frac{\mathrm{A}}{\mathrm{p}-\mathrm{A}}-\frac{2 \mathrm{TA} \lambda}{\mathrm{p}-\mathrm{A}}\right)\right\}+\mathrm{Q}_{1}^{2}\left(\frac{\mathrm{A} \lambda}{(\mathrm{p}-\mathrm{A})^{2}}\right) \\
\left.-\mathrm{AT}+\mathrm{A} \lambda \mathrm{T}^{2}\right) \\
C^{*}=\mathbf{5 7 4 . 8 6 7 4}
\end{gathered}
$$

\section{Sensitivity analysis}

The effects of changes in the values of the parameters $\theta, C_{1}, C_{2}, p, A$ and $T$ on the optimal total inventory cost, stock level and backlog level derived by the proposed method. The sensitivity analysis is performed by changing the value of each of the parameters by $50 \%,-25 \%, 25 \%$, and $50 \%$, taking one parameter at a time and keeping the remaining six parameter unchanged. On the basis of the results shown in the Table 1, the following observations can be made. 
Inventory Model for Deteriorating Items with no Shortages

Table 1: Sensitivity analysis

\begin{tabular}{|c|c|c|c|}
\hline Parameter & \% change & \% change in Q1 & \% change in C* \\
\hline \multirow{4}{*}{$\theta$} & -50 & -43.3155 & -2.7012 \\
& -25 & -20.7778 & -1.6191 \\
& 25 & 22.2470 & 1.7786 \\
& 50 & 43.7343 & 4.1065 \\
\hline \multirow{4}{*}{$\mathrm{C}_{1}$} & -50 & 0.2381 & -32.2158 \\
& -25 & 0.7444 & 0.1734 \\
& 25 & 1.7568 & 64.7036 \\
& 50 & 2.2630 & 96.9407 \\
\hline \multirow{4}{*}{$\mathrm{C}_{2}$} & -50 & -1.0507 & 2.6935 \\
& -25 & -1.0517 & 2.6987 \\
& 25 & -1.0537 & 2.7089 \\
& 50 & -1.0547 & 2.7141 \\
\hline \multirow{4}{*}{$\mathrm{P}$} & -50 & -340.8994 & -3.1910 \\
& -25 & -312.8212 & -1.5502 \\
& 25 & -268.4715 & 1.8616 \\
& 50 & -252.3682 & 3.5149 \\
\hline \multirow{4}{*}{$\mathrm{A}$} & -50 & -1.3210 & 8.6141 \\
& -25 & -1.3003 & 7.9243 \\
& 25 & -1.2601 & 6.6498 \\
$\mathrm{~T}$ & 50 & -1.2406 & 6.0617 \\
\hline & -50 & -725.2282 & -9.2045 \\
& -25 & -758.1321 & -4.2179 \\
& 25 & -823.9297 & 1.0838 \\
& 50 & -856.8296 & 5.8514 \\
\hline
\end{tabular}

It is observed from Table that the solution is sensitive to change all the parameter $\theta, C_{1}, C_{2}, A, T, p$.

\section{Conclusion}

It is obvious that, in the business world the demand rate cannot be considered as a fixed. Hence the researcher allows the demand rate and the deterioration time of the item to be stochastic and follows exponential distribution. Based on this a new inventory model for deteriorating without shortages is design and experimented with the empirical data.

\section{REFERENCES}

1. Bhanu Priya Dash, Trailokyanath Singh and Hadibandhu Pattnayak, An inventory model for deteriorating items with exponential declining demand and time-varying holding cost, American Journal of Operations Research, 4 (2014) 1-7.

2. K.Hesham Alfares, Inventory model with stock-level dependent Demand rate and variable holding cost, International Journal in Production Economics, 108 (2007) 259-265.

3. N.K.Kaliraman, R.Raj, S.Chandra, and H.Chaudhry,An EPQ inventory model for deteriorating items with weibull deterioration under stock dependent demand, International Journal of Scientific Technology Research, 4 (1) (2015). 
P.Mariappan, M.Kameswari and M.Antony Raj

4. S.Khanra and K.S.Chaudhuri, A production-inventory model for a deteriorating item with shortage and time dependent demand, Yugoslav Journal of Operations Research, 21 (2011) 29-45.

5. D.Kiransinh, Rathod and Pravin H. Bhathawala, Inventory model with stock dependent demand rate variable ordering cost and variable holding cost, IJSIMR, 2 (2014) 637-641.

6. Kuo-Lung Hou, Yung-Fu Huang and Li-Chiao Lin, An inventory model for deteriorating items with stock-dependent selling rate and partial backlogging under inflation, African Journal of Business Management, 5 (10) (2011) 3834-3843.

7. P.K.Lakshmi Devi and M.Maragatham, An inventory model with three rates of production and time dependent deterioration rate for quadratic demand rate, International Journal Fuzzy Mathematical Archive, 6 (1) (2015) 99-103.

8. M.Maragatham, and P.K.Lakshmi Devi, An inventory model for non- instantaneous deteriorating items under conditions of permissible delay in payments for $n$-cycles, International Journal Fuzzy Mathematical Archive, 2 (2013) 49-57.

9. P.Mariappan, Operations Research Methods and Applications, New century book house, $2^{\text {nd }}$ Edition, (2002).

10. Manisha Pal and Sujan Chandra, A periodic review inventory model with stock dependent demand, permissible delay in payment and price discount on backorders, Yugoslav Journal of Operations Research, 24(1) (2014) 99-110.

11. Sachin Kumar, Pawan Kumar and Manju Saini, An order level inventory model for deteriorating items with quadratic demand rate and Variable holding cost, IJSRET, (2012) 253-263.

12. G.P Samanta and Ajanta Roy, A deterministic inventory model of deteriorating items with two rates of production and shortages, Tamsui Oxford Journal of Mathematical Sciences, 20(2) (2004) 205-218.

13. G.P.Samanta and Ajanta Roy, A production inventory model with deteriorating items and shortages, Yugoslav Journal of Operations Research, 14(2) (2004) 219-230.

14. Sandeep Kumar, A.K.Vasishtha and Ashima Gupta, A production control inventory model for continuous deteriorating items with shortages and two different rates of productions, International Journal of Education and Science Research Review, 4(1) (2014).

15. C.K.Sivashankari and S.Panayappan, Production inventory model for two-level production with deteriorative items and shortages, The International Journal of Advanced Manufacturing Technology, 76 (2015) 9-12.

16. Sanjai Sharma, An improved two-warehouse partial backlogging inventory model for deteriorating items under conditionally permissible delay in payment, IJARCET, 4(11) (2015) 453-364. 
Inventory Model for Deteriorating Items with no Shortages

17. D.Sharmila, and R.Uthayakumar, An inventory model with three rates of production rate under stock and time dependent demand for time varying deterioration rate with shortages, IJAEMS, 2(9) (2016) 453-461.

18. S. Shital Patel, Production inventory model for deteriorating items with different deterioration rates under stock and price dependent demand and shortages under inflation and permissible delay in payments, Global Journal of Pure and Applied Mathematics, 13 (2017) 3687-3701. 